\title{
Systematische Erfassung von Einflussfaktoren für das Additive Tooling von Spritzgusswerkzeugen
}

\author{
Steffen Schrock ${ }^{1,2 *}$, Andreas Proksch ${ }^{1}$, Simon Rapp ${ }^{2}$, Stefan Junk ${ }^{1}$, Albert \\ Albers ${ }^{2}$ \\ ${ }^{1}$ Hochschule Offenburg | Labor Rapid Prototyping \\ 2 KIT - Karlsruher Institut für Technologie | IPEK - Institut für Produktentwicklung \\ ${ }^{*}$ Korrespondierender Autor: \\ Steffen Schrock \\ Hochschule Offenburg \\ Klosterstr. 14 \\ 77723 Gengenbach \\ Telefon: 07803 9698-4473 \\ Mail: steffen.schrock@hs-offenburg.de
}

\section{Abstract}

Additive tooling is a quick and cost-effective way of producing injection molded products and high fidelity prototypes using the injection molding process. As part of product development, additive tooling is integrated into a complex process. A lack of design and application knowledge represents a barrier in its use. The present work shows how a Design-Structure-Matrix (DSM) can be used to systematically record and analyze influencing factors and their interrelationships. A systematic literature search is carried out to identify the factors and relationships.

\section{Keywords}

Additive Tooling, Injection Molding, Rapid Tooling, Design-StructureMatrix 


\section{Motivation}

Das Additive Tooling (AT) stellt eine schnelle und kostengünstige Möglichkeit dar, um seriennahe Prototypen im Spritzgussverfahren zu produzieren [1-4]. Allerdings wird der Einsatz des Additive Tooling durch eine komplexe Prozessabfolge und fehlendes Anwendungs- und Konstruktionswissen erschwert [5-7]. Bei der additiven Fertigung von Spritzgusswerkzeugen, können die auf konventionelle Werkzeuge (zerspanen, erodieren) ausgelegten Konstruktionsregeln nur eingeschränkt angewandt werden. Auch das strikte Befolgen des Design for Additive Manufacturing (DfAM) ist nur bedingt zielführend, da beim Additive Tooling von Spritzgusswerkzeugen die Anforderungen an den Spritzguss- und AMProzess simultan betrachtet werden müssen $[6,8]$. Zudem werden bereits mit der Gestaltung des Produktes die Anforderungen an das Spritzgusswerkzeug und den Spritzgussprozess gesetzt, wodurch eine Betrachtung des Gesamtprozesses erforderlich wird. Prinzipiell wird dadurch ein erweitertes Regelwerk erforderlich, welches als Design for Additive Tooling (DfAT) bezeichnet werden könnte. Eine für den Produktentwicklungsprozess durchgängige Vorgehensweise zur Nutzung von AT steht in der heutigen Entwicklungspraxis ebenfalls noch nicht zur Verfügung. Durch die Nutzung von strukturierenden Methoden und Tools soll eine Wissensbündelung und -vermittlung ermöglicht werden.

\section{Stand der Forschung}

\subsection{Technische Betrachtung}

Additive Tooling (AT) beschreibt einen Anwendungsbereich der additiven Fertigung (Additive Manufacturing, AM), bei dem eine schnelle Herstellung von Formen bzw. Werkzeugen erfolgt [1]. Im Gegensatz zum konventionellen Tooling (KT) wird beim Additive Tooling das Werkzeug schichtweise aufgebaut, wodurch die Realisierung komplexer Geometrien möglich ist. Gängige additive Verfahren zur Fertigung von Werkzeugeinsätzen sind Material-Jetting ((MJ), z.B. PolyJet (PJ), MultiJet Modelling (MJM)), Stereolithografie (SLA) und Digital Light Processing (DLP). Diese Verfahren beruhen auf dem Prinzip der Photopolymerisation und bieten eine, für additive Verfahren, gute Oberflächenqualität und Fertigungsgenauigkeit. Prinzipiell führt die Verwendung der AT-Formeinsätze aufgrund der schlechten Wärmeleitfähigkeit zu einer verlängerten Zykluszeit im Spritzgussprozess [7].

Wie bei den meisten AM-Verfahren besteht der Vorteil, nahezu beliebige Geometrien zu fertigen. So lassen sich unter Anderem beliebige Verläufe und Querschnitte für Kühlkanäle vorsehen. Neben einem konturnahen Verlauf der Kühlkanäle, lässt sich auch ein an die Kontur angepasster Querschnitt realisieren. Verschiedene Untersuchungen zeigen, dass dadurch eine effektivere Wärmeableitung erreicht werden kann als mit Kühlkanälen mit kreisförmigem Querschnitt [9-11]. Da die Kanäle für eine effektive Wärmeabfuhr, bedingt durch die schlechte Wärmeleitfähigkeit der Polymerwerkzeuge, nah an der Kavität liegen müssten, würde sich dadurch jedoch eine zu geringe Wandstärke ergeben, die dem Druck durch die Kunststoffschmelze meist nicht mehr standhalten kann [12]. Ein Versagen der ATFormeinsätze ist die Folge. Ahrens et al. [13] beschreiben eine Vorgehensweise, bei der mithilfe von „Heat Flux Canals“ eine effizientere Kühlung erreicht werden kann. Dazu wird das Werkzeug additiv als Schale hergestellt und mit einem wärmeleitfähigen Material aufgefüllt. Anschließend wird die an bestimmten Stellen sehr dünne Schale überschliffen, sodass das Füllmaterial dort an der Oberfläche liegt und sich so ein Wärmeleitkanal bildet.

Kovács [14] hat sich mit der Vorhersage und dem konstruktiven Ausgleich der Schwindung innerhalb der Werkzeugkonstruktion beschäftigt. So sollen durch Simulation Schwindungen im Voraus erkannt werden, die dann wiederum im CAD-Modell des Werkzeuges konstruktiv berücksichtigt werden. Kumar et al. [15] haben Digital-ABS auf die Schwindungseigenschaften hin untersucht und ein integriertes Modell zum Schwindungsausgleich in der Konstruktionsphase entworfen. Laut Palmer und Colton [16], sowie Mendible et al. [17] stellen 
konstruktive Ausführungen des Werkzeugs, wie Formschrägen und Hinterschnitte, auch einen wichtigen Aspekt für die Standzeit eines Werkzeugs dar. Gerade das Auswerfen der Spritzlinge geht noch einmal mit einer hohen mechanischen Belastung einher, die durch eben diese Ausführungen beeinflusst wird. Griffiths et al. [18] haben außerdem die Bauteilorientierung während der additiven Fertigung als eine Eigenschaft ausgemacht, die sich ebenfalls auf die Lebensdauer auswirkt.

Schuh et al. [19] haben die Machbarkeit und Prozesssicherheit von einfachen Schieberwerkzeugen untersucht. Dabei wurden Standzeiten von rund 100 Zyklen erreicht. Je nach Anwendungsfall sind aber auch längere Standzeiten möglich [20]. Schuh et al. [21] stellen ein Modell vor, dass als Entscheidungshilfe für bestimmte Maßnahmen in Konstruktion oder Prozess für das effiziente Abkühlen der Kunststoffformeinsätze dienen soll. Dabei soll zwischen Maßnahmen wie z. B. Nutzung von Kühlkanälen, Druckluftkühlung oder Materialzusätzen gewählt werden können.

\subsection{Wirtschaftliche Betrachtung}

Bagalkot et al. [22] zeigen durch eine Analyse von Angeboten aus der Industrie, dass selbst bei einer einfachen Werkzeuggeometrie, die wenige aufwändige Bearbeitungsschritte benötigt, die Herstellungskosten im PolyJet-Verfahren nur $50 \%$ der Kosten eines herkömmlichen Stahlwerkzeugs betragen. Dazu beträgt die Lieferzeit lediglich zwei Tage, während bei Stahl- oder Aluminiumwerkzeugen mit 14 Tagen zu rechnen ist. Kampker et al. [7] vergleichen die Verwendung mehrerer PolyJet- und SLA-Materialien mit konventionell gefertigten Spritzgusswerkzeugen. Bei der Mehrheit der Materialien liegt die Kostenersparnis bei der Werkzeugherstellung zwischen $35 \%$ und $75 \%$. Eine Gegenüberstellung der Werkzeugherstellungskosten, auf Basis eines Fallbeispiels, ergab eine Ersparnis bei additiver Werkzeugherstellung von ca. $30 \%$ bis $40 \%$ (Digital ABS und Rigur gegenüber Aluminium). Die Spritzgussproduktionskosten bei Anwendung der AT-Formeinsätze liegen dabei jedoch ca. 50 \% höher im Vergleich zu den Aluminiumformeinsätzen. Wesentlicher Grund dafür ist die deutlich längere Zykluszeit bei Verwendung der Polymerwerkzeuge. Die Gesamtkostenersparnis liegt dennoch bei ca. $10 \%$ bis $15 \%$ [20].

Storsanden et al. [23] geben an, dass bis zu zehn Mal mehr Entwicklungsiterationen in sechs Monaten möglich sind. Weiterhin haben sie für das Reaction Injection Moulding (RIM) eine Kostenersparnis von bis zu $98 \%$ ermittelt. Der Trend zur Mass Customization könnte sich damit auch beim Spritzgießen etablieren. Im Beispiel von Tosello et al. [24] verringern sich die Stückkosten um $80 \%$ bei einer Losgröße von 100 Stück. Auch die Produktionszeit des Werkzeugs verringert sich um bis zu zwei Drittel, sowie die Werkzeugkosten um $91 \% \mathrm{im}$ Vergleich zu einem konventionell hergestellten Werkzeug. Die Autoren stellen außerdem ein Modell vor, mit dem der Stückpreis ermittelt und verglichen werden kann, um über das geeignete Verfahren zu entscheiden.

\section{Handlungsbedarf \& Forschungsproblem}

Der Stand der Forschung zeigt, dass Forscher bereits unterschiedlichste Teilgebiete im Forschungsfeld „Additive Tooling“ untersucht haben (technische und wirtschaftliche Eigenschaften, Prozessparameter, Möglichkeiten der Simulation etc.). Dadurch konnten bereits spezielle Einflussfaktoren, Attribute und Wirkmechanismen identifiziert und beschrieben werden. Diese Erkenntnisse stammen jedoch aus einer fragmentierten Forschung mit unterschiedlichen Schwerpunkten und Abstraktionsgraden. Zudem kommt das Problem der Aktualität der verfügbaren Informationen. Durch sich wandelnde Rahmenbedingen, wie bspw. neue AM-Verfahren, Materialen usw. müssen Wissenselemente allerdings in entsprechenden Abständen hinterfragt und gegebenenfalls aktualisiert werden. Weiterhin zeigen die Untersuchungen, dass der Wechsel vom konventionellen, hin zum 
additiven Tooling eine weitreichende strukturelle Anpassung des Prozessablaufes nötig macht. Da Entwicklungsprozesse individuell sind, müssen bestimmte Elemente und deren Beziehung zueinander unter Umständen situationsspezifisch beurteilt werden.

Daraus ergibt sich die Notwendigkeit eines ganzheitlichen Ansatzes, der die Modellierung des Gesamtprozesses sowohl in der praktischen Entwicklung als auch in der wissenschaftlichen Diskussion unterstützt. Das Forschungsziel besteht in der Identifikation relevanter Prozesselemente und -abläufe aus den Bereichen Spritzguss und Additive Tooling, sowie der anschließenden Entwicklung einer methodischen Unterstützung. Besonders den Anwendern des Additive Tooling soll ein zielgerichtetes Vorgehensmodell an die Hand gegeben werden, welches sie bei Entscheidungs-, Konstruktions- und Anwendungsprozessen unterstützt. Die aus der Zielstellung abgeleitete Forschungsfrage lautet:

Wie können die komplexen, disziplinübergreifenden Prozesse des Additive Tooling in einer anwendergerechten Form abgebildet und der Analyse zugänglich gemacht werden?

\section{Methodik \& Vorgehensweise}

Zunächst wird eine Prozessbeschreibung des Additive Tooling vorgenommen und mit Experten diskutiert. Dadurch werden die zur Herstellung von Spritzgussprodukten notwendigen Hauptelemente (Verfahren, Prozess, Produkt) definiert, zu denen Informationen beschafft werden. Zudem dient diese Beschreibung der vereinfachten Darstellung des Gesamtprozesses und dem Schaffen eines gemeinsamen Systemverständnisses (Abschnitt 4.1).

Darauf aufbauend werden entsprechende Suchkriterien abgeleitet, welche die direkte Beziehung von mindestens zwei Elementen enthalten (z.B. „Additive Tooling \& PolyJet“ oder „Additive Tooling \& Injection Molding“). Anschließend werden durch eine systematische Literaturrecherche (Abschnitt 4.2) Einflussfaktoren, Prozessparameter und wechselseitige Beziehungen auf einer breiten Basis ermittelt und ausgewertet. Die Ergebnisse der Recherche werden schließlich in einer Design-Structure-Matrix (DSM) modelliert und analysiert (Abschnitt 4.3). Der Fokus liegt dabei zunächst nicht auf der vollständigen und genauen Abbildung des Realprozesses, sondern auf der Systematik zur Modellierung.

\subsection{Prozessschaubild "Additive Tooling"}

Zweck des Additive Tooling (AT) im Spritzguss ist die Herstellung von Spritzgussprodukten oder Spritzgussprototypen. Dazu wird durch additive Fertigungsverfahren ein Spritzgusswerkzeug (i.d.R. ein Formeinsatz, welcher in eine Stammform eingesetzt wird) hergestellt. Zu Beginn erfolgt die Wahl eines Fertigungsverfahren zur Werkzeugherstellung. Additives und konventionelles Tooling besitzen unterschiedliche Verfahrensprofile und eignen sich je nach Anforderung nicht gleich gut. Neben Faktoren welche die Verfahren nach "besser“ oder "schlechter" einteilen lassen gibt es, besonders auf Seiten des AT viele KO-Kriterien, wonach eine Nutzung dieser Werkzeuge ausgeschlossen werden kann (z.B. zu hohe Schließkräfte, Wärmebelastung, Einspritzdruck, usw.).

Bei der additiven Herstellung eines Werkzeuges bzw. Formeinsatzes werden bereits der Großteil seiner Eigenschaften festgelegt. Dabei spielen das Additive Verfahren, das AMMaterial, die AM-Anlage, die Geometrie des zu fertigenden Werkzeuges, sowie die spezifischen AM-Prozessparameter (z.B. Bauteilorientierung, Schichtstärke, Druckmodus usw.) eine entscheidende Rolle. Durch den Spritzgussprozess wird das Spritzgussmaterial im Spritzgusswerkzeug letztlich zum Spritzgussprodukt verarbeitet. Die Eigenschaften des Produkts hängen dabei wesentlich vom Spritzgussmaterial, der gewählten Prozessparameter und auch vom genutzten Werkzeug ab. Das zu fertigende Spritzgussprodukt gibt seinerseits durch seine Gestalt und spezifischen technischen und wirtschaftlichen Anforderungen von Beginn an gewisse Restriktionen an das Werkzeug, das Spritzgussmaterial und den 
Spritzgussprozess vor, wodurch ein komplexes Gebilde von Wechselbeziehungen entsteht (Bild 1).

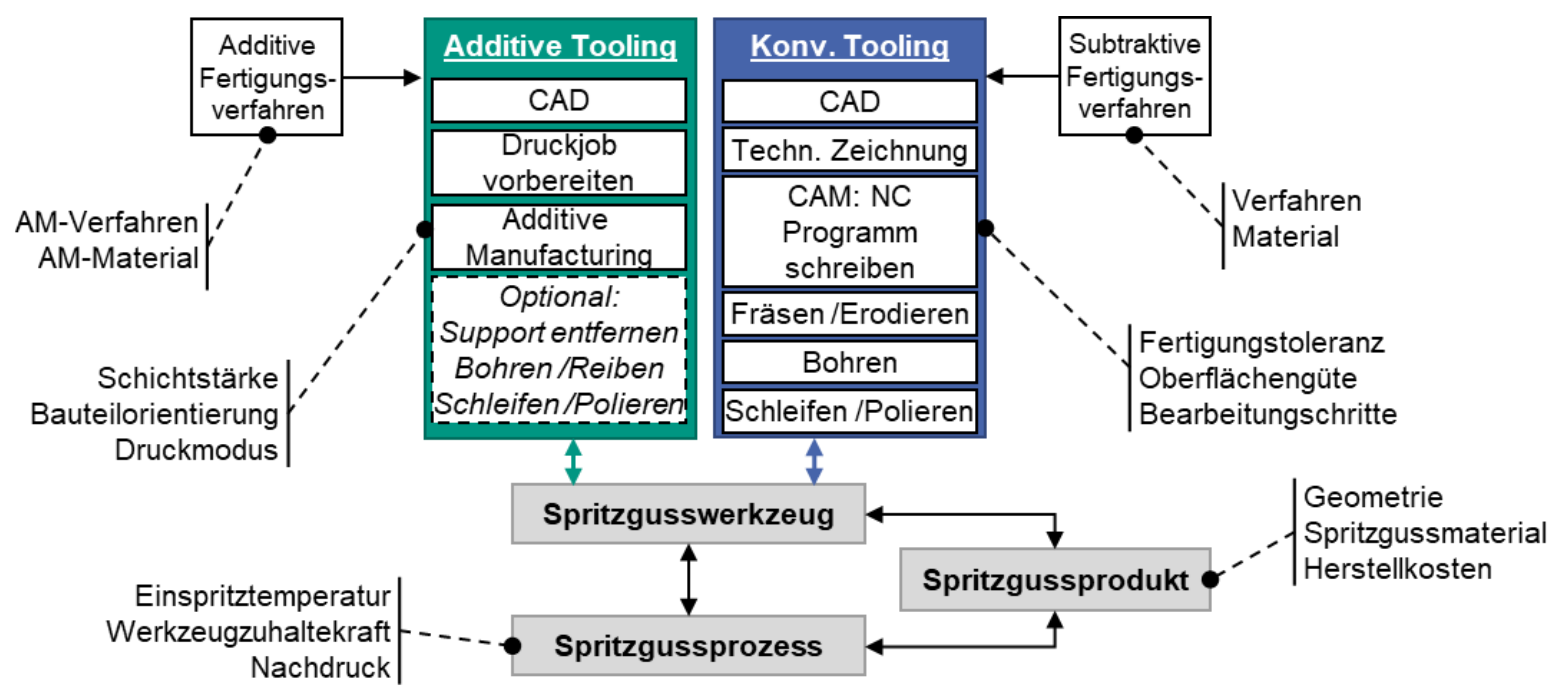

Bild 1: Wechselbeziehung von Kernelementen beim Spritzgießen mit beispielhaften Sub-Elementen

Eine semi-strukturierte Befragung von Experten $(n=5)$ zum Kenntnisstand über Additive Tooling zeigt wesentliche Wissenslücken und Unterstützungsbedarfe in verschiedenen Phasen des Entwicklungsprozesses, beginnend bei der Wahl des geeigneten AM-Verfahren bis hin zur praktischen Umsetzung und Anwendung der AT-Werkzeuge. Zusammenfassend werden folgende Kernfragen aus Expertensicht formuliert und den betreffenden Projektphasen auf Anwendungsebene zugeordnet (Bild 2). Eine eigene Durchführung eines Projektes zur additiven Fertigung und Nutzung eines AT-Werkzeuges bestätigt diese Bedarfe.

Wissenslücke zum Additive Tooling: $\quad$ AT-Projektphase

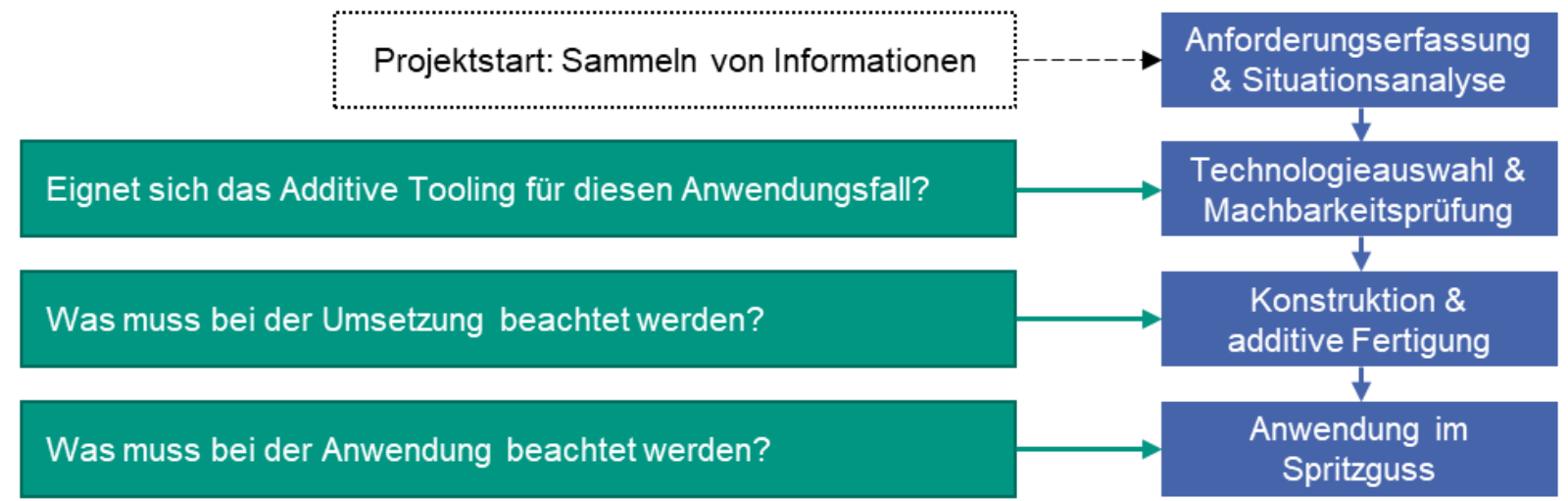

Bild 2: Projektphasen auf Anwendungsebene des AT für die eine Unterstützung erzielt werden soll

\subsection{Literaturrecherche}

Zur systematischen Literaturrecherche wird nach Fachliteratur und wissenschaftlichen Publikationen gesucht, welche additive Verfahren untersuchen, die mit Photopolymerisation arbeiten. Dazu zählen die Stereolithographie (SLA), das Digital Light Processing (DLP), sowie das Material-Jetting (MJ, z.B. PolyJet (PJ), MultiJet Modelling (MJM)). Einerseits wurde die Anwendbarkeit dieser Verfahren für das Additive Tooling bereits nachgewiesen und andererseits lassen sich die Verfahren hinsichtlich vieler Gesichtspunkte verhältnismäßig gut untereinander vergleichen. Für die Recherche werden Veröffentlichungen zwischen 1995 bis 
Oktober 2020 miteinbezogen. Dabei wurden mehrere Kombinationen deutsch- und englischsprachiger Begriffe aus den Bereichen Spritzguss und additiver Fertigung unter Beachtung differenzierter Schreibweisen verwendet. Ergebnis der Recherche sind insgesamt 199 Titel (Stand: 15. Oktober 2020), die unter diese Suchbegriffe und Kriterien fallen. Von 115 Titeln konnte der Volltext zur weiteren Untersuchung genutzt werden (Bild 3).

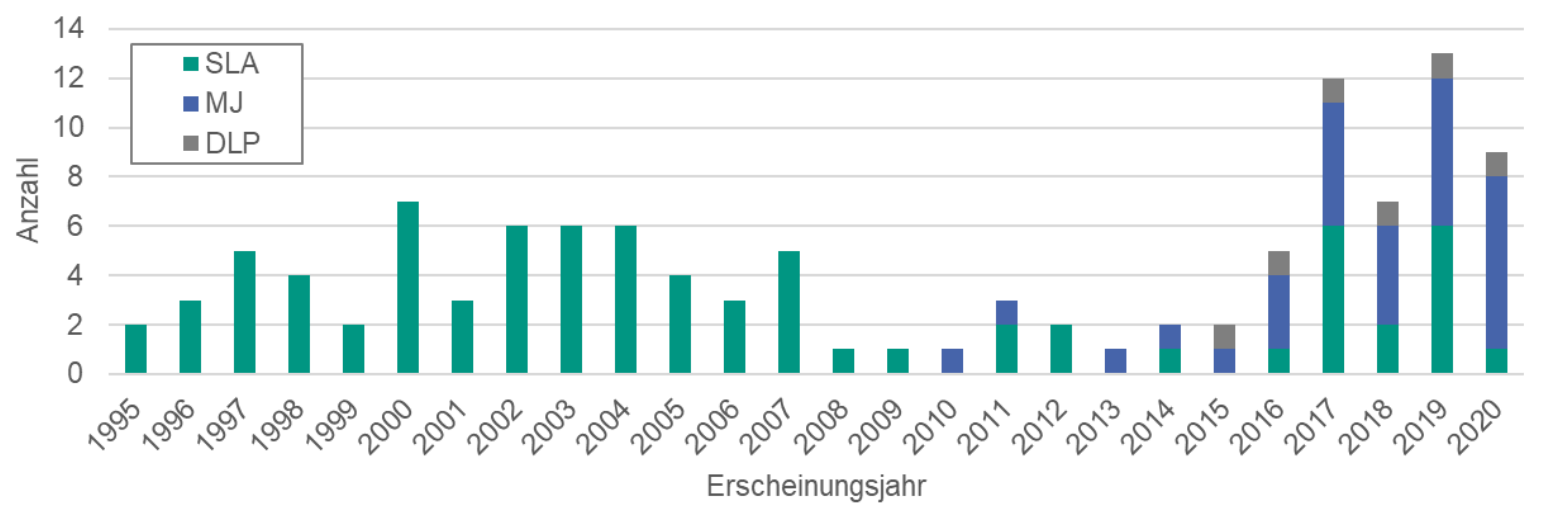

Bild 3: Verteilung der genutzten Quellen nach Fertigungsverfahren und Erscheinungsjahr

Eine chronologische Betrachtung der gefundenen Publikationen zeigt eine stetige Zunahme innerhalb der letzten fünf Jahren. Gründe dafür sind mutmaßlich in der Verbesserung bestehender, als auch dem Hinzukommen neuer Verfahren und Materialien zu finden. In den gesichteten Veröffentlichungen werden vorwiegend praktische Versuche und Experimente beschrieben, bei denen durch Additive Tooling gefertigte Spritzgussformeinsätze auf der Spritzgussmaschine getestet werden. Oft sind diese Versuchsreihen durch vorherige Simulationen unterstützt worden. Die Schwerpunkte der Untersuchungen liegen meist auf den mechanischen und thermischen Eigenschaften der Werkzeuge und teilweise auch auf den mechanischen Eigenschaften und der Qualität der Spritzgussprodukte. In einigen Fällen erfolgten die Versuche unter Variation der AM-Verfahren, AM-Materialien und Spritzgussmaterialien. Die Vorgehensweise zur Konstruktion und additiven Fertigung der Werkzeuge bzw. Formeinsätze wird ebenfalls oft geschildert. Derzeit überwiegen noch Publikationen zum Stereolithographie-Verfahren, wobei MultiJet-Modeling (MJ) und Digital Light Processing (DLP) in den jüngeren Veröffentlichungen stark vertreten sind. Als Spritzgussmaterialien sind sowohl teilkristalline als auch amorphe Polymere verwendet worden, wobei Polypropylen und ABS zusammen mehr als die Hälfte ausmachen (Bild 4).
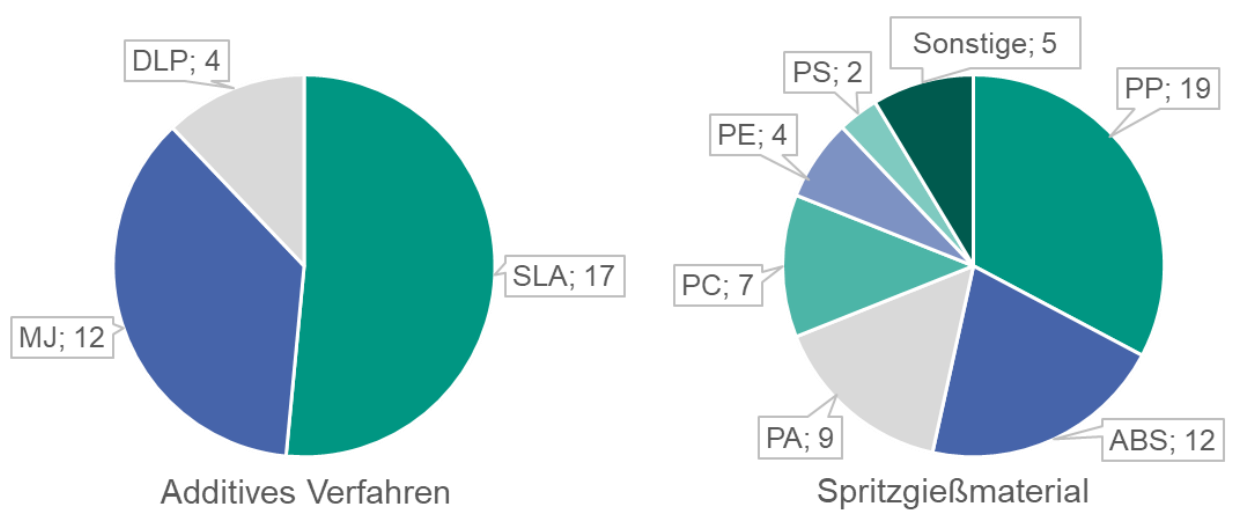

Bild 4: Art und Anzahl der AM-Verfahren und Spritzgussmaterialien aus den praktischen Versuchsreihen 


\subsection{Modellierung der Design-Structure-Matrix (DSM)}

Die recherchierten und selektierten Quellen werden analysiert und Faktoren mit Wechselwirkung als Wissenselement gespeichert. Zur übersichtlichen Aufbereitung und Analyse der Informationen wird als Methode eine numerische Design-Structure-Matrix (DSM) gewählt. Die DSM-Elemente werden dabei mit einem Faktor zwischen (0, -1] (schwache bis starke negative Korrelation) bis $(0,+1]$ (schwache bis starke positive Korrelation) in Beziehung gesetzt. Somit wird nicht nur die Richtung der Korrelation, sondern auch eine Gewichtung vorgenommen, was insbesondere die Rolle eines Elements bei der Änderungsfortpflanzung beeinflusst. Weiterhin kann prinzipiell zwischen quantitativen (z.B. physikalisch oder mathematisch) und qualitativen Zusammenhängen unterschieden werden. Die Quelle jeder Beziehung wird ebenfalls dokumentiert (Bild 5). Als Beispiel führt eine Erhöhung des Nachdruckes zu weniger bzw. geringeren Einfallstellen (qualitativ) aber auch zur Notwendigkeit einer höheren Schließkraft (quantitativ).

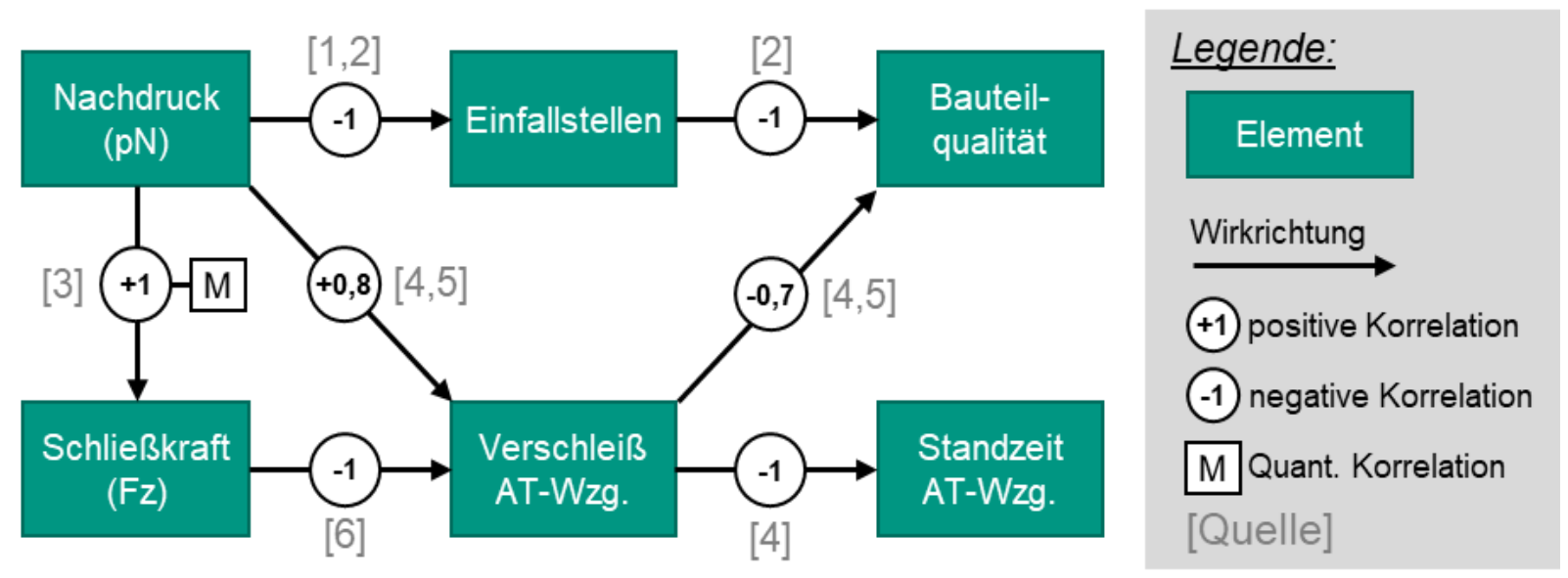

Bild 5: Prinzip der DSM-Modellierung anhand beispielhafter AT-Einflussfaktoren in grafischer Darstellung

\section{Ergebnisse \& Diskussion}

\subsection{Tool zur Auswertung der DSM}

Die Erfassung und Analyse der Daten erfolgt über ein selbst entwickeltes VBA-Tool aus MS-Excel Basis, welches verschiedene Funktionen erfüllt:

- Erfassung der Elemente, Beziehungen und Quellen für konventionelles und additives Tooling in einer eigenständigen DSM

- Auswertung der Aktivität und Kritikalität der Elemente

- Durchführen einer Pfadanalyse zur Auswertung und Darstellung der Änderungsfortpflanzung

- Partitionierung der DSM

- Überlagerung der AT-DSM und KT-DSM zum direkten Vergleich der DSM-Elemente und Beziehungen

Die Auswertung hinsichtlich passender Kennzahlen erfordert eine möglichst vollständige und präzise Abbildung des Realprozesses, da es ansonsten zu Fehlinterpretationen kommen kann. Dies betrifft nicht nur das Vorhandensein oder Fehlen wichtiger Elemente, sondern auch die Richtung und Gewichtung der gesetzten Korrelationen. Unter anderem können folgende Auswertungen an der DSM vorgenommen werden (Tabelle 1): 
Tabelle 1: Kennzahlen zur Auswertung der DSM

\begin{tabular}{|l|l|l|}
\hline Kennzahl & Berechnung & Bedeutung \\
\hline Aktivsumme (AS) & Bildung der Zeilensumme & $\begin{array}{l}\text { Zeigt ausgehende Abhängigkeiten } \\
\text { als Maß für die Einflussnahme }\end{array}$ \\
\hline Passivsumme (PS) & Bildung der Spaltensumme & $\begin{array}{l}\text { Zeigt eingehende Abhängigkeiten } \\
\text { als Maß für die Beeinflussung }\end{array}$ \\
\hline Aktivität (Akt) & AS / PS & Rolle bei Änderungsfortpflanzung \\
\hline Kritikalität (Krit) & AS * PS & Gesamtgewicht eines Elements \\
\hline
\end{tabular}

Bislang konnten 61 relevante Elemente identifiziert und 111 Zusammenhänge für das Additive Tooling (aus der systematischen Literaturrecherche), sowie 90 Verbindungen für das konventionelle Tooling (aus einschlägiger Fachliteratur) mittels Design-Structure-Matrix modelliert werden. Da die Präzisierung der Korrelationen noch weiterer Untersuchung bedarf, wird an dieser Stelle keine Auswertung der DSM vorgenommen, sondern ein erster Auszug der DSM vorgestellt (Bild 6).

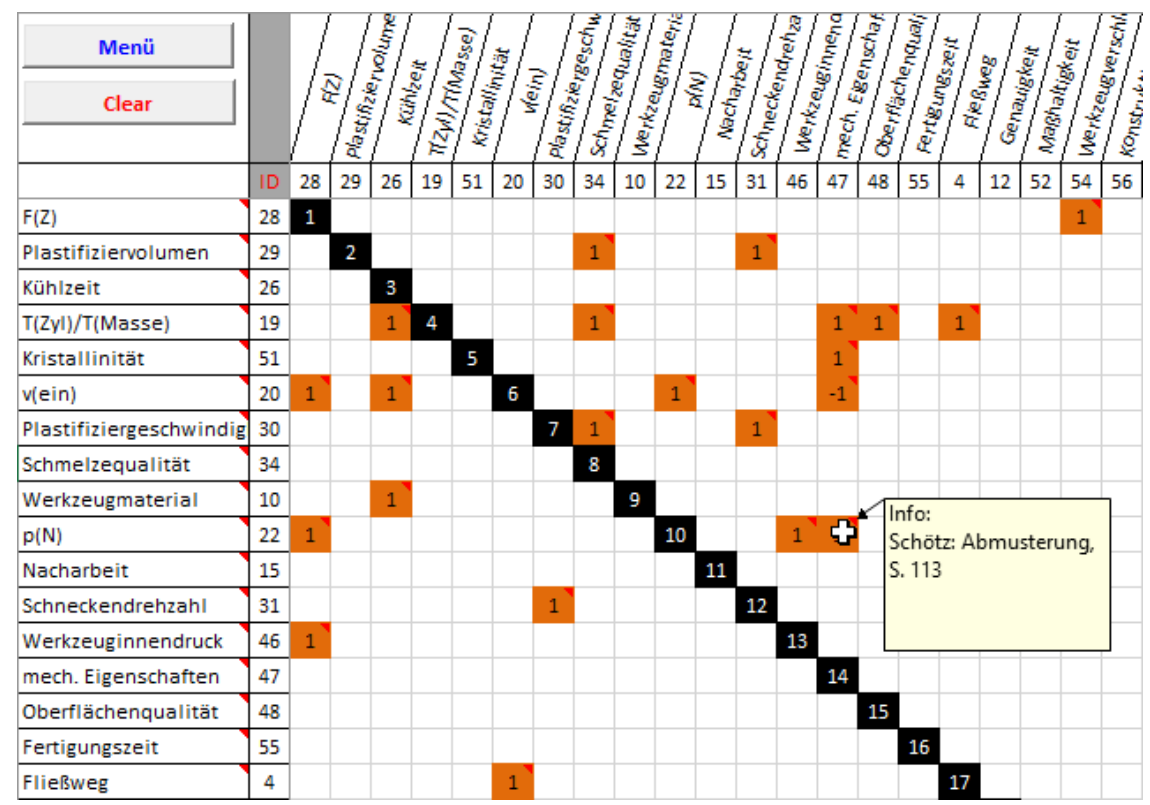

Bild 6: Auszug aus der erzeugten DSM mit Darstellung einer Quelle zur entsprechenden Korrelation

Durch eine Überlagerungsfunktion kann verglichen werden, ob die gleichen Voraussetzungen für das konventionelle und additive Tooling gelten, oder ob und in welchen Punkten sich diese unterscheiden (Bild 7). Anwender des Additive Tooling, welche mit dem konventionellen Prozess vertraut sind, können sich so zielgerichtet auf abweichende Elemente konzentrieren.

Konv. Tooling

\begin{tabular}{|l|l|l|l|l|l|}
\hline $\mathbf{4}$ & $\mathbf{1}$ & $\mathbf{2}$ & $\mathbf{3}$ & $\mathbf{4}$ & $\mathbf{5}$ \\
\hline $\mathbf{1}$ & & $\mathrm{X}$ & & $\mathrm{O}$ & \\
\hline 2 & & & $\mathrm{O}$ & $\mathrm{X}$ & \\
\hline 3 & & & & & $\mathrm{X}$ \\
\hline 4 & & & & & \\
\hline 5 & & & & & \\
\hline
\end{tabular}

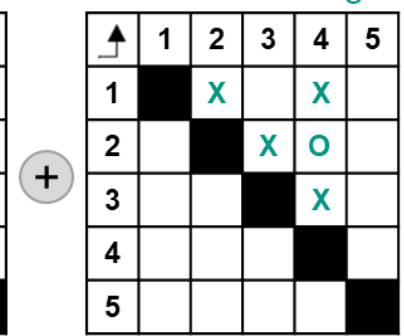

AT-KT-Korrelation

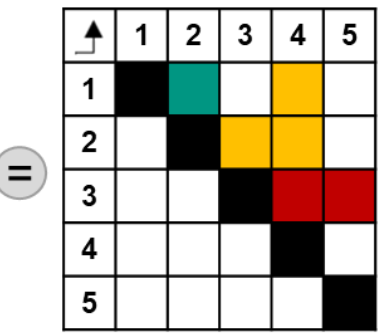

\begin{tabular}{|c|l|}
\hline $\mathbf{X}$ & Starke Korrelation \\
\hline $\mathbf{O}$ & Schwache Korrelation \\
\hline & Keine Korrelation \\
\hline
\end{tabular}

Identisch

Korrelation vorhanden

Wesentlicher Unterschied

Bild 7: Überlagerung der KT-DSM und AT-DSM zum Verfahrensvergleich 


\subsection{Modell zur systematischen Erfassung von Einflussfaktoren}

Durch das vorgestellte Modell lässt sich der komplexe Gesamtprozess des Additive Tooling zur Herstellung von Spritzgussprodukten bzw. Prototypen modellieren, dokumentieren und einer Analyse, sowie einer Wissensspeicherung und -vermittlung zugänglich machen (Bild 8). Durch die Analysefunktionen der DSM lassen sich die Auswirkungen von Änderungen im Gesamtprozess erkennen und nachverfolgen. Um dem individuellen Charakter von Produktentwicklungsprozessen und sich ändernden Randbedingungen Rechnung tragen zu können, kann die DSM beliebig erweitert und angepasst werden.

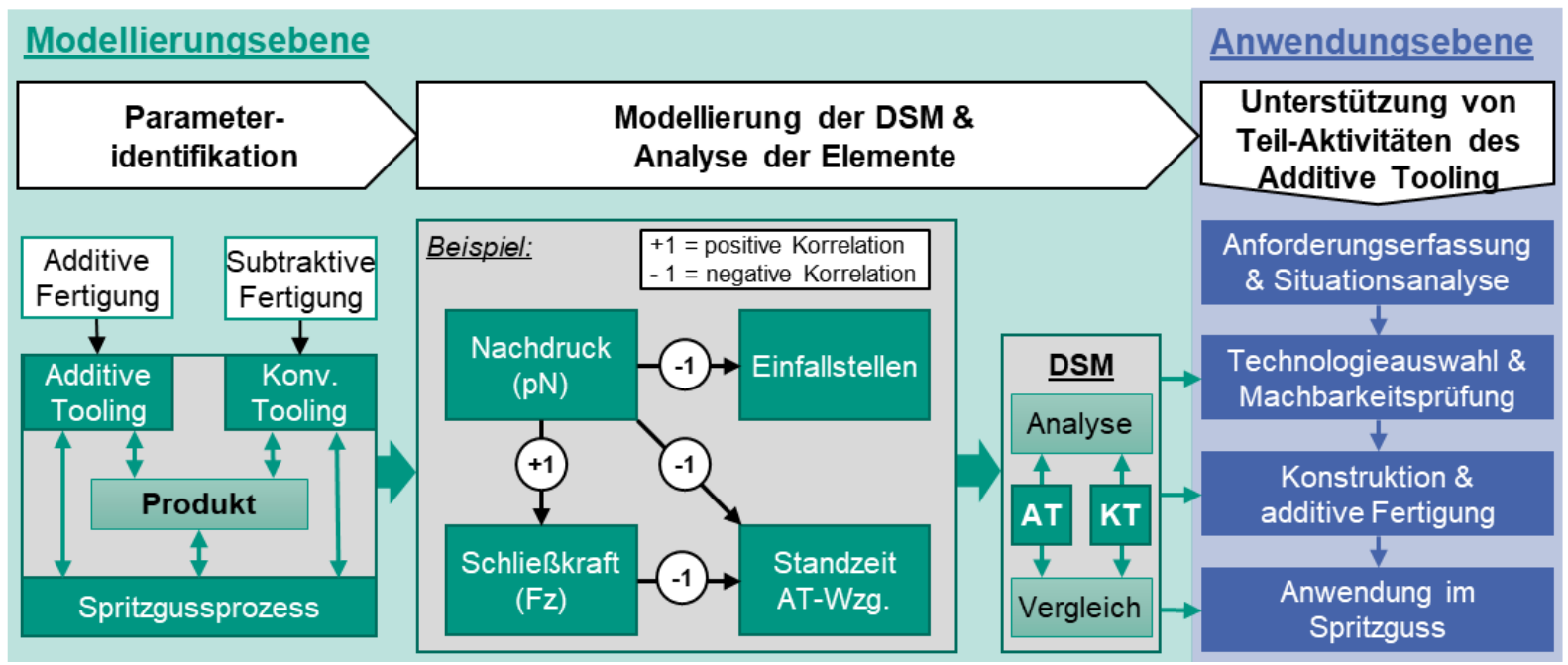

Bild 8: Modell zur systematischen Erfassung von Einflussfaktoren per DSM zur Unterstützung verschiedener TeilAktivitäten des Additive Tooling

\section{Zusammenfassung \& Ausblick}

Die fehlende Verfügbarkeit eines ganzheitlichen Ansatzes zur Auslegung und Anwendung des Additive Tooling stellt eine große Herausforderung für Anwender dar. Die durchgeführte Literaturstudie zeigt die Komplexität der Prozesslandschaft und das disziplinübergreifende Fachwissen, welches zur Implementierung und Anwendung von Additive Tooling notwendig ist. Durch die systematische Erfassung der Prozesselemente mittels DSM lassen sich die Wirkzusammenhänge beschreiben, veranschaulichen und einer Analyse zugänglich machen. Dadurch können kritische Parameter und Wissenslücken identifiziert und dokumentiert werden. Die somit erzeugte Wissensbasis kann auf Anwendungsebene von der Technologieauswahl bis zum Einsatz im Spritzguss unterstützen.

Entlang des Entwicklungsprozesses sollen neben der DSM zukünftig weitere, situationsspezifisch unterstützende Methoden zur Verfügung gestellt werden. Der erarbeitete Ansatz der DSM-Modellierung wird im nächsten Schritt im Rahmen eines Workshops und anhand eines Entwicklungsprojektes in der Industrie evaluiert und die DSM-Elemente und deren Beziehungen werden präzisiert.

\section{Literaturverzeichnis}

[1] Gebhardt, Andreas: Additive Fertigungsverfahren: Additive Manufacturing und 3D-Drucken für Prototyping Tooling - Produktion. 5., neu bearbeitete und erweiterte Auflage. München: Hanser, 2016.

[2] Kampker, Achim et al.: Potential analysis of additive manufacturing technologies for fabrication of polymer tools for injection moulding - A comparative study. In: 2018 IEEE International Conference on Advanced Manufacturing (ICAM), 2018, S. 49-52.

[3] Feldhusen, Jörg (Hrsg.); Grote, Karl-Heinrich (Hrsg.): Pahl/Beitz Konstruktionslehre: Methoden und Anwendung erfolgreicher Produktentwicklung. Berlin, Heidelberg: Springer Vieweg, 2013. 
[4] Schrock, Steffen et al.: Potentiale des Additive Tooling zur Steigerung der Effizienz und Agilität von Validierungsaktivitäten in der frühen Phase der Produktentwicklung. In: 18. Gemeinsames Kolloquium Konstruktionstechnik 2020: Nachhaltige Produktentwicklung: KT 2020, S. 259-270.

[5] Nagahanumaiah; Subburaj, K.; Ravi, B.: Computer aided rapid tooling process selection and manufacturability evaluation for injection mold development. In: Computers in Industry 59 (2008), Nr. 2, S. 262-276.

[6] Gebhardt, Andreas; Kessler, Julia; Schwarz, Alexander: Produktgestaltung für die Additive Fertigung. Müchen: Hanser, 2019.

[7] Kampker, Achim; Alves, Bruno; Ayvaz, Peter: Technological and Economic Comparison of Additive Manufacturing Technologies for Fabrication of Polymer Tools for Injection Molding. In: Almeida, Henrique A.; Vasco, Joel C. (Hrsg.): Progress in Digital and Physical Manufacturing. Cham: Springer International Publishing, 2020 (Lecture Notes in Mechanical Engineering), S. 28-39.

[8] Mitterlehner, Thomas: Auslegung und Charakterisierung additiv gefertigter Formeinsätze für Spritzgießwerkzeuge. Universität Linz, Institut für Polymer-Spritzgießtechnik und Prozeßautomatisierung. Dissertation, 2020.

[9] Wu, Tong et al:: A Framework for Optimizing the Design of Injection Molds with Conformal Cooling for Additive Manufacturing. In: Procedia Manufacturing 1 (2015), S. 404-415.

[10] Altaf, Khurram et al.: Determining the effects of thermal conductivity on epoxy molds using profiled cooling channels with metal inserts. In: Journal of Mechanical Science and Technology 30 (2016), Nr. 11, S. 49014907.

[11] Jahan, Suchana; El-Mounayri, Hazim: A Thermomechanical Analysis of Conformal Cooling Channels in 3D Printed Plastic Injection Molds. In: Applied Sciences 8 (2018), Nr. 12, S. 2567.

[12] Rodríguez, Jorge: Use of Additive Manufacturing (AM) to Fabricate Injection Molding Inserts. In: Proceedings of the 14th LACCEI International Multi-Conference for Engineering, Education, and Technology: "Engineering Innovations for Global Sustainability": Latin American and Caribbean Consortium of Engineering Institutions, 2016.

[13] Ahrens, C. H.; Ribeiro Jr, A. S.; Beal, V. E.: Heat flux canals (HFC) technique: an alternative to cool down stereolithography moulds. In: Journal of the Brazilian Society of Mechanical Sciences and Engineering 25 (2003), Nr. 3.

[14] Kovács, József Gábor: Construction of Pre-Deformed Shapes for Rapid Tooling in Injection Molding. In: Macromolecular Symposia 239 (2006), Nr. 1, S. 259-265.

[15] Kumar, Sagar; Singh, Amit Kumar: Volumetric shrinkage estimation of benchmark parts developed by rapid tooling mold insert. In: Sādhanā 45 (2020), Nr. 1.

[16] Palmer, A. E.; Colton, J. S.: Failure mechanisms in stereolithography injection molding tooling. In: Polymer Engineering \& Science 40 (2000), Nr. 6, S. 1395-1404.

[17] Mendible, Gabriel Antonio; Rulander, Jack A.; Johnston, Stephen P.: Comparative study of rapid and conventional tooling for plastics injection molding. In: Rapid Prototyping Journal 23 (2017), Nr. 2, S. 344-352.

[18] Griffiths, C. A. et al.: Micro-stereolithography tools for small-batch manufacture of polymer micro-parts. In: Proceedings of the Institution of Mechanical Engineers, Part B: Journal of Engineering Manufacture 226 (2012), Nr. 4, S. 708-721.

[19] Schuh, Günther et al.: Feasibility and Process capability of polymer additive injection molds with slide technology. In: Procedia CIRP 93 (2020), S. 102-107.

[20] Kampker, Achim et al.: Direct Polymer Additive Tooling - Verwendung von Polymerwerkzeugen für den Einsatz im Kleinserien Spritzguss. In: Kynast, Michael; Eichmann, Michael; Witt, Gerd (Hrsg.): Rapid.Tech + FabCon 3.D - International Trade Show + Conference for Additive Manufacturing. München: Carl Hanser Verlag GmbH \& Co. KG, 2018, S. 45-62.

[21] Schuh, Günther et al.: Towards Temperature Control Measures for Polymer Additive Injection Molds. In: Procedia CIRP 93 (2020), S. 90-95.

[22] Bagalkot, Anurag et al.: A methodology for setting the injection moulding process parameters for polymer rapid tooling inserts. In: Rapid Prototyping Journal 25 (2019), Nr. 9, S. 1493-1505.

[23] A. L. Storsanden; M. Våle ; R. M. C. Ratnayake: Use of additive manufacturing for polymer tooling: Case study from reaction injection molding. In: 2017 IEEE International Conference on Industrial Engineering and Engineering Management (IEEM), 2017, S. 1607-1610.

[24] Tosello, Guido et al.: Value chain and production cost optimization by integrating additive manufacturing in injection molding process chain. In: The International Journal of Advanced Manufacturing Technology 100 (2019), 1-4, S. 783-795. 